\title{
How to Improve Efficiency of Telecom Energy
}

\author{
Changling Zhang, Lu Xu and Jiangang Liu \\ Energy Product Line, ZTE, Shenzhen 518057, China
}

Received: November 09, 2015 / Accepted: November 27, 2015 / Published: January 31, 2016.

\begin{abstract}
High OPEX (operation expenditure), pollution and complicated environment are challenges which telecom operators have to conquer to improve energy efficiency. Integration and flexibility are tendencies for telecom energy solution development, also the keys to improve energy efficiency. With plentiful design and implementation experience, the author provides a three-dimensional energy matrix model to design a perfect energy system, and some practical measures of integration and flexibility are also shared from mechanical to electrical, from single site to whole network.
\end{abstract}

Key words: Energy efficiency, three-dimension energy matrix model, optimization practices.

\section{Three Major Challenges in Existing Telecom Energy Solution}

For a typical scenario of traditional telecom energy, high fuel consumption, low efficiency, large footprint and high consumption of temperature control are common issues. These issues pushed traditional energy solution evolvement, from DG (disel generator) solution to battery-DG hybrid solution, then to solar hybrid solution. Customers are benefited by these improvements. However there are still three major challenges.

First one is high OPEX (operation expenditure). DG is still important resource for outdoor sites, and expense related to DG is a large part of OPEX. Besides DG, O \& M (operation \& maintenance) expense and site rental are also high. Second one is pollution. Carbon emission and noise due to fuel consumption caused much concerns. The reasons why we can not deploy more solar hybrid sites are high cost and large footprint of solar site. Third one is complicated environment. The environment is changing at different area and different period. For example, public grid supply is various between town and suburb; End users are increasing year by year; Solar irradiation varies in

Corresponding author: Changling Zhang, solution supervisor, research field: energy solution. days and seasons. A flexible system with low expansion and upgrading cost must be supplied to protect investment.

To improve energy system efficiency, some ways must be found to solve these three challenges.

\section{Tendencies and Energy Model}

There are already many practices and studies to solve the above mentioned challenges. They can be summarized as integration and flexibility.

Integration means structures and/or functions are integrated together. For example, outdoor solution replaced indoor solution, less space and less energy consumption; especially a single control center manages all the energy elements, low cost and fast. Higher power density and smarter control will contribute to less OPEX and less pollution.

Flexibility is the way to react to the changing conditions. Few or low cost to expand or upgrade is key issue to protect investment and decrease CAPEX (capital expenditure).

Integration and flexibility are keys to conquer the three challenges. Many measures were already taken to optimize the energy solution following the two tendencies. However, these measures are scattered and partial. An energy model to describe where and how to improve the energy efficiency will be helpful to 


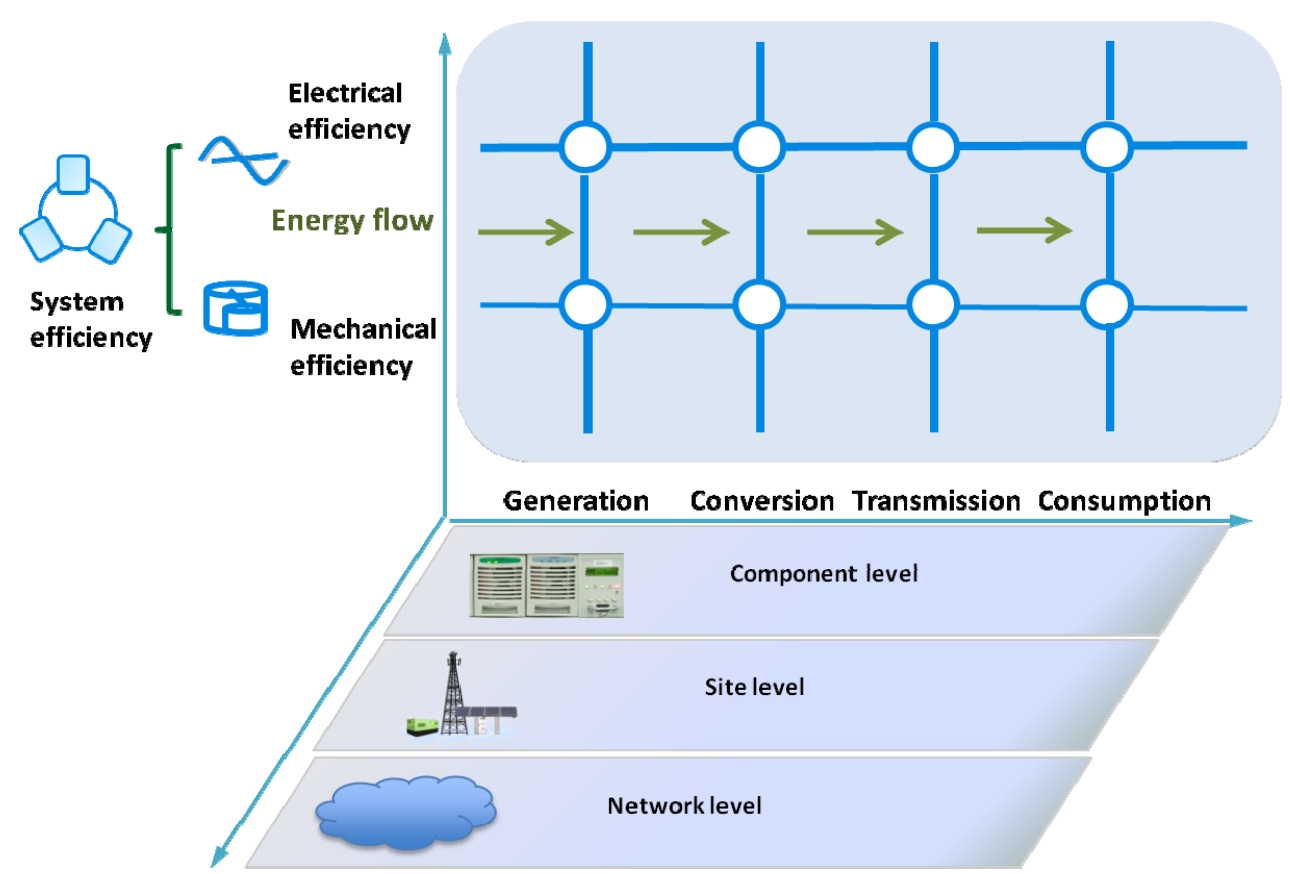

Fig. 1 Three dimension energy matrix model.

optimize the energy system comprehensively and continuously.

With more than 20 years experience of research \& development and practices in ZTE corporation, we proposed three-dimension energy matrix model (Fig. 1).

At the upper side, the energy matrix $[1,2]$ is the basic model to describe an energy system. In $\mathrm{X}$ axis, system energy flows in four processes, from generation, conversion, transmission to consumption. This describes the four core processes how energy comes, converts and goes. In $\mathrm{Y}$ axis, energy system is described with electrical and mechanical components, from which we can improve the system efficiency. The energy matrix gives us a blueprint of an energy system, telling how energy changes and how can we make optimization. To improve electrical and mechanical efficiency in these four processes will achieve high system efficiency.

What is the content in $\mathrm{Z}$ axis? In practice, according to the actual conditions and finance plan, these optimizations are implemented at three levels, component, site and network level. Different manufacturer may focus on different level. From three levels operators can find good products or solutions. Since system thinking is more important, nowadays there are more innovations at site level and network level.

With this model, we will easily find where and how to make progress.

\section{Telecom Energy Solution Optimization Practices}

ZTE energy team has been devoted himself to telecom energy solution optimization. We would like to share some verified experience at these three levels.

\subsection{Practices at Component Level}

Higher component efficiency and smaller dimension are main directions for optimization. In generation process, except public grid, DG and solar are two popular energy resources.

We introduced variable speed DG. The controller adjusts engine to run at variable speed, low speed at low load ratio decrease the fuel consumption. Averagely $10 \%$ expense saving can be achieved compared with fixed speed DG. Even $40 \%$ can be saved at low load ratio (Fig. 2). 

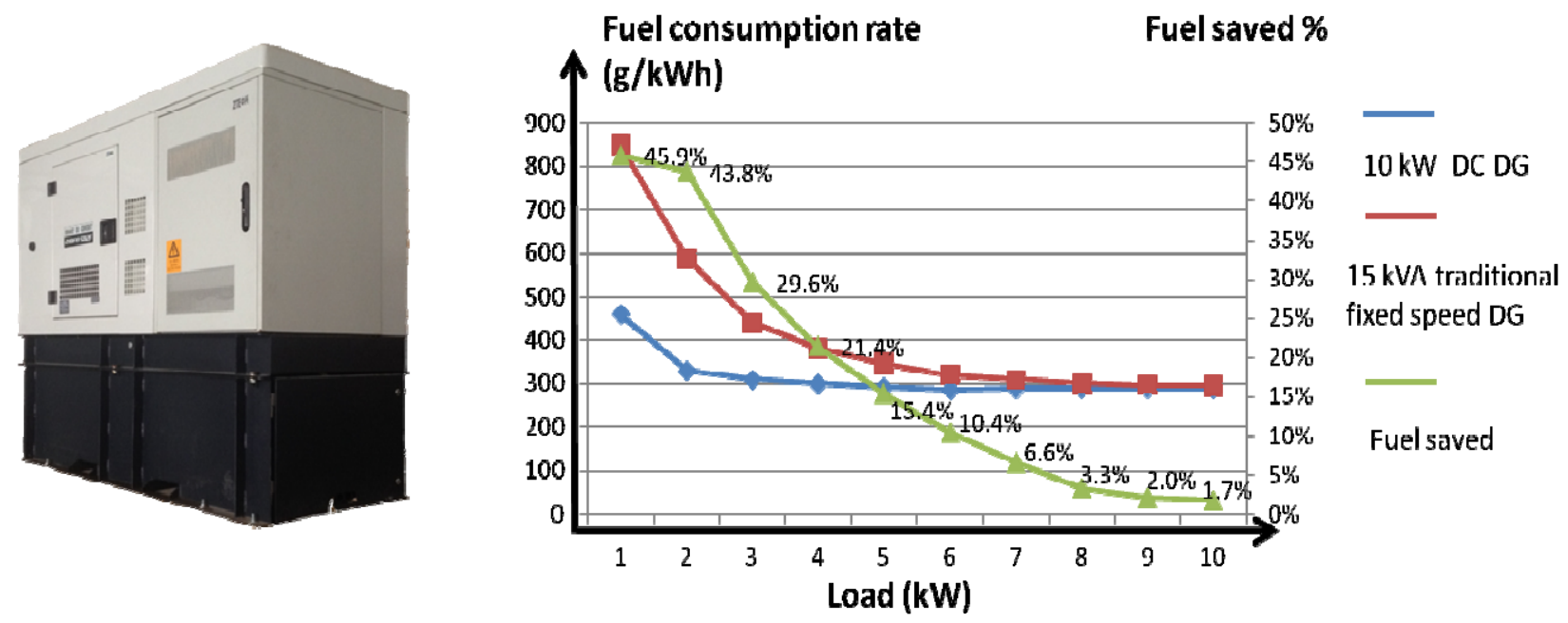

Fig. 2 Fuel consumption comparison between variable speed DG \& fixed speed DG.

Green energy especially solar energy is used widely. 98\% power unit with MPPT (maximum power point tracking) and $15.3 \%$ silicon solar panel help to improve efficiency too.

In conversion process, rectifier is most concerned component which contributes greatly to the system efficiency. Its efficiency grows from $88 \%$ to $96 \%$ in latest years, about $1,700 \mathrm{kWh}$ can be saved annually for $2 \mathrm{~kW}$ telecom site. And rectifiers with $98 \%$ efficiency will be of commercial value in the coming years. Hibernation function will be the basic function which will cut down the self consumption to $2 \mathrm{~W}$.

In consumption process, cooling energy for battery is of high cost and battery space is also a challenge for some applications. New types of battery are deployed except traditional VRLA (valve regulated lead acid) and gel battery.

Heat proof battery can save more cooling energy, its excellent performance at high temperature $(1,800$ @ $45{ }^{\circ} \mathrm{C} 60 \%$ DOD (deep of discharge)) need only nature cooling.

Lithium battery is lighter and deep DOD application, suitable for roof top or limited space application. It is fast charging, suitable for multiple outage scenarios.

\subsection{Practices at Site Level}

Recently, it is difficult to make great step forward when focusing on single components. In latest five years, more concerns were on site level solutions.

At site level, comprehensive optimization is focused, covering both electrical and mechanical, more than one single process. We will share some innovative measures and opinions.

\subsubsection{Single Control Center}

Among these innovations, most important one is single control center.

One single control center instead of multiple centers has been the popular solution. Most control orders are from the single CSU (centralized supervision unit). That means faster and smarter control.

The single CSU can not only manage all the required parts and parameters (such as grid, solar, battery, DG, fuel and environment parameters), but also manage the energy elements in a more specific way (Fig. 3).

For example, it can arrange flexible charging schedule during different grid tariffs. During outage period, when grid price is low, the center will enable large charge current as $0.2 \mathrm{C}$, while small charge current say $0.05-0.1 \mathrm{C}$ when grid price is high. That will decrease electricity expense about $10 \%$.

With energy priority optimization, higher fuel consumption is achieved because of larger DG charging current to both load and battery. And through PV priority plan, more solar energy can be used, too. 


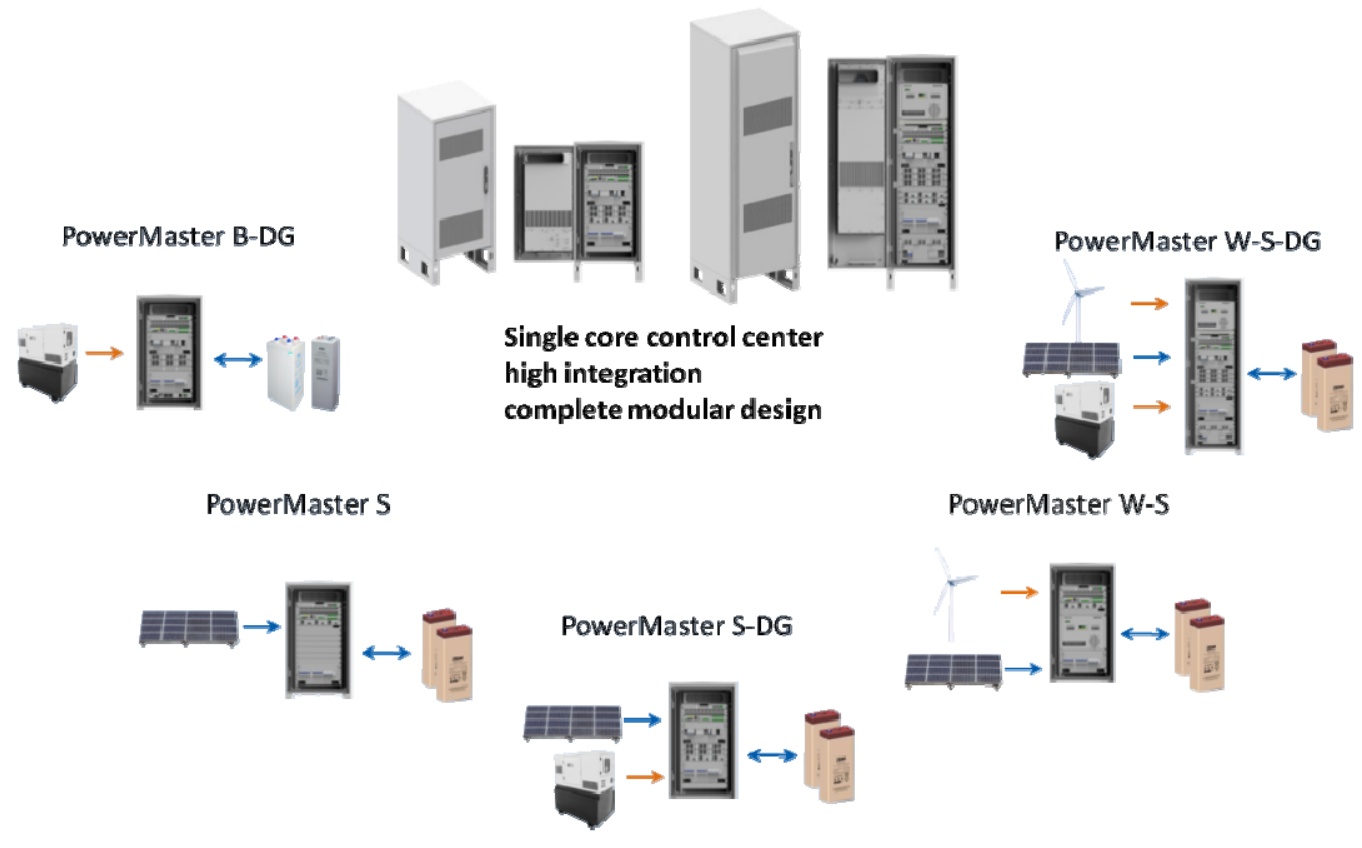

Fig. 3 Single control center model.
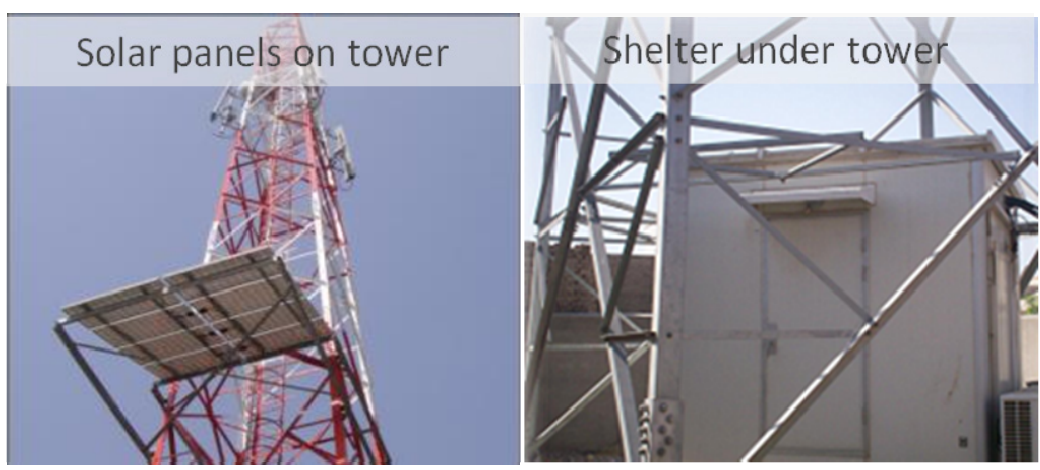

Fig. 4 Space saving practices.

And the single control center should support flexible combinations.

With modular design concept for other energy elements, the system should support capacity expansion and solution evolution in an easy way and cost-effective way.

With ZTE PowerMaster solution, only some new modules or parts are added in and software is upgraded during these upgrading processes. This will help reusing existing equipment and cut down the TCO (total cost of ownership).

Furthermore, site sharing expansion must be considered, too. Because the land is so limited and sharing solution can achieve higher efficiency.
ZTE PowerMaster and PowerMaster+ [3] solution can support the expansion from one operator to at least three operators, and also support upgrading from battery hybrid solutions to solar hybrid solutions.

\subsubsection{Space Saving Innovations}

More integrated structure means less space. We integrated ATS (automatic transfer switch), fuel tank and DG together, then integrated DC power system further more. Floor space can be saved about $50 \%$ amazingly. Engineering cost is saved at the same time because of less cable and excavation.

Space availability is always a limitation to deploy solar solution. Solar on tower and shelter or cabinet beneath tower (Fig. 4), the two solutions can promote 
solar application in a limited area.

\subsubsection{Energy Saving for Temperature Control}

For indoor sites, smart cooling solution will cut down the cooling consumption greatly, which takes use of nature air cooling.

And most indoor sites can be transformed to outdoor site, saving more cooling energy by DC air conditioner in cabinet. Partition cooling in the same cabinet enables higher temperature for telecom equipment saving energy further.

\subsection{Practices at Network Level}

If we want to share network resources, a remote monitoring system is necessary. The RMS (remote monitoring system) can help to cut down network maintenance cost and support continuous optimization. It should have three important characters.

Firstly, all the energy elements can be easily accessed to the RMS anywhere and in any way. All the necessary components and all the necessary parameters should be monitored.

Secondly, it can help to enhance energy efficiency management. Based on energy efficiency monitoring and analysis, we can know whether the energy system is healthy and make right decision to optimize the combination and parameters. For example, the battery charging time, charging current, whether to remove DG, whether to add or decrease quantity of rectifiers.

Finally, maintenance supporting helps cut down maintenance cost. By comparing the running data with historical data and benchmark, the RMS system can give maintenance plan such as refueling, DG maintenance, battery replacement and solar module replacement. Active maintenance helps cut down the on-site labor cost and improve $\mathrm{O} \& \mathrm{M}$ efficiency.

\section{Conclusions}

High OPEX, pollution, complicated environment are major challenges in existing telecom energy system, which required integration and flexibility. In practice, we suggest the energy model, energy matrix at three levels (component, site and network), which will outline where and how to make improvement for energy solution. And we shared some optimization measures and ideas at three levels such as single control center, DC DG, RMS rules, etc.

\section{Future Outlook}

Ninety-eight percent high efficiency rectifier seems the future choice in the coming years. However, more optimization or innovation will emerge at site level and network level. Solutions and measures support site sharing have great potential since cost saving and land limitation. RMS should be integrated with technologies from other industries, such as big data mining and IOT (Internet Of Things).

\section{References}

[1] Xiaoguang, L. 2010. "Energy Matrix Application in Renewable Energy Solution." COMMUNICATIONS WEEKLY, August 23.

[2] Xiaoguang, L., and Jiangang, L. 2011. "Energy Matrix Creates Future of Green Telecommunication." ZTE Technologies 8 (April): 10-2.

[3] Yun, W., and Jiankai, G. 2015. "Sharing Low Carbon Communication, Cobuilding Green Future." COMMUNICATIONS WEEKLY, April 17. 